\title{
DCE-MRI and parametric imaging in monitoring response to neoadjuvant chemotherapy in breast carcinoma: a preliminary report
}

\author{
Anjna Sharma ${ }^{1 A, B, E, F}$, Sanjiv Sharma ${ }^{1 A, D}$, Shikha Sood ${ }^{1 A, D}$, Rajeev K. Seam ${ }^{2 A}$, Mukesh Sharma ${ }^{2 B, E, F}$, Vikas Fotedar ${ }^{2 E}$ \\ 'Department of Radiodiagnosis, Indira Gandhi Medical College, Shimla, H.P., India \\ ${ }^{2}$ Department of Radiotherapy, Regional Cancer Centre, Indira Gandhi Medical College, Shimla, H.P., India
}

\section{Abstract}

\begin{abstract}
Purpose: Neoadjuvant chemotherapy is recommended in patients with locally advanced breast cancer. Dynamic contrast-enhanced magnetic resonance imaging (DCE-MRI) enables evaluation of the tumour neovasculature that occurs prior to any volume change, which helps identify early treatment failures and allows prompt implementation of second-line therapy.
\end{abstract}

Material and methods: We conducted a prospective study in 14 patients with histopathologically proven breast cancer. DCE-MRI data were acquired using multisection, T1-weighted, 3D vibe sequences with fat suppression before, during, and after IV bolus injection $(0.1 \mathrm{mmol} / \mathrm{kg}$ body weight, Gadoversetamide, Optimark). Post-processing of dynamic contrast perfusion data was done with the vendor's Tissue $4 \mathrm{D}$ software to generate various dynamic contrast parameters, i.e. Ktrans, Kep, Ve, initial area under the time signal curve (IAUC), apparent diffusion coefficient (ADC), and enhancement curve. Patients underwent MRI examinations at baseline, and then after two cycles, and finally at completion of chemotherapy.

Results: Based on Sataloff criteria for pathological responses, four patients out of 14 were responders, and 10 were non-responders. At the 2nd MRI examination, IAUC was significantly smaller in responders than in non-responders $(p=0.023)$. When the results of the first and second MRI examinations were compared, Kep decreased from baseline to the second MRI $(p=0.03)$ in non-responders and in responders $(p=0.04)$. This change was statistically significant in both groups. The ADC values increased significantly in responders from baseline to the third MRI $(p=0.012)$.

Conclusions: In our study, IAUC and ADC were the only parameters that reliably differentiated responders from non-responders after two and three cycles of chemotherapy.

Key words: DCE-MRI, neoadjuvant chemotherapy, response evaluation, carcinoma of the breast.

\section{Introduction}

Neoadjuvant chemotherapy (NACT), i.e. administration of chemotherapy before surgical treatment, has now become the standard initial treatment for locally advanced breast cancer. NACT has the advantages of rendering inoperable patients operable, so they can then become candidates for breast-conserving surgery. NACT also allows in situ assessment of response to a particular chemotherapeutic regimen, leading to a timely switch to an alternative, effective regimen in non-responders.

Physical examination, mammography, and ultrasonography (USG) can be used to evaluate tumour response after chemotherapy, but they have several limitations, e.g. the presence of fibrosis and necrosis hinder the accuracy of clinical examination. Similarly, dense breast,

Correspondence address:

Mukesh Sharma, MD, Department of Radiotherapy, Regional Cancer Centre, Indira Gandhi Medical College, Shimla, H.P., India 171001,

e-mail:muk00008@gmail.com

Authors' contribution:

A Study design · B Data collection · C Statistical analysis · D Data interpretation · E Manuscript preparation · F Literature search · G Funds collection 
architectural distortion, calcifications, and fibrous reaction to chemotherapy hinder the interpretation of mammography, whereas multifocal and in situ disease evaluation is difficult with USG.

MRI is superior to physical examination, ultrasound, and mammography in response evaluation, and has high accuracy in predicting pathological complete remission after NACT in patients with breast cancer. It also has the advantage of differentiating fibrosis from tumour tissue. The presence of dense breast does not affect the accuracy of MRI, and the identification of multifocal and multicentre disease is possible with this modality.

Dynamic contrast-enhanced MRI (DCE-MRI) utilises the changes in tumour vasculature for imaging. Neoangiogenesis, i.e. formation of new blood vessels, is an early change in tumour development. The permeability of newly formed vessels allows contrast material to leak into the interstitium.

The transfer constant, $K^{\text {trans }}$, describes the transendothelial transport of contrast medium by diffusion from the vascular space to the tumour interstitium, and provides a measure of vascular permeability. In tumours, $K^{\text {trans }}$ values are high. Over a period lasting typically several minutes to hours, the contrast agent diffuses back into the vasculature. This is described by the rate constant or $K^{e p} . V_{e}$ describes the extracellular extravascular space. These parameters are related by the following equation: $K^{e p}=K^{\text {trans }} / V_{e}$.

In patients with breast carcinoma who receive NACT, a significant reduction of up to a third has been shown with regard to both $K^{\text {trans }}$ and $K^{e p}$. In addition, an increase in $V_{e}$ of nearly a third has been shown in non-responders [1]. A change in $K^{\text {trans }}$ greater than $40 \%$ has been considered as the threshold for definitive response [2]. However, the results of different studies show discrepancies, with some studies showing little or no decrease in $K^{\text {trans }}$ or $K^{e p}$ following neoadjuvant chemotherapy [3]. In fact, one small study (Yu et al.) performed among 29 patients, who were scanned after one cycle of chemotherapy, showed that early tumour size change is a better response predictor than $K^{\text {trans }}$ and $K^{\text {ep }}[4]$.

Semi-quantitative parameters can be calculated from enhancement curves, including the onset time - from injection to the appearance of contrast in the tissues, maximum signal intensity, gradient or rate of contrast uptake and washout, and initial area under the time signal curve (IAUC). Diffusion-weighted MRI is another technique in which the diffusivity of water molecules is weighted in different tissues. Quantitative images of the diffusion coefficient or more exactly of the apparent diffusion coefficient (ADC) are then obtained. In malignant tissues, high cellular density and lipophilic cell membranes are present, leading to low values of ADC. After chemotherapy, apoptosis of malignant cells occurs. Moreover, increased membrane permeability and increased extracellular space cause much higher ADC values after chemotherapy than before it. This change occurs earlier than changes in other parameters. However, due to a limited number of studies, further evaluation of these techniques is needed.

\section{Material and methods}

\section{Study setting and study population}

The present study was conducted at our institution among patients hospitalised in surgery and oncology departments from June 2013 to June 2014. The study was performed with the approval of the institution's Ethics Committee. Written consent was obtained from all patients or patient's attendants.

Inclusion criteria:

1) cytological or histological confirmation of primary breast carcinoma

2) breast tumour $>3 \mathrm{~cm}$ in diameter or stage IIIA or stage IIIB disease,

3) no previous surgical or chemotherapeutic treatment,

4) age between 18 and 70 years,

5) haematological values - haemoglobin $>10 \mathrm{~g} / \mathrm{dl}$, platelets $>100 \times 10^{9} / 1$, neutrophils $>1.5 \times 10^{9} / 1$,

6) normal liver function tests (LFT),

7) glomerular filtration rate $(\mathrm{GFR})>30 \mathrm{ml} / \mathrm{min} / 1.73 \mathrm{~m}^{2}$, using the MDRD formula.

\section{Sampling procedure}

All consecutive patients who met the inclusion criteria were included in the study. MRI examinations were performed, the first before the standard neoadjuvant chemotherapy regimen, the second after two cycles of chemotherapy, and the third after completion of NACT if NACT was given for more cycles.

\section{Technique}

MRI studies were performed using a 1.5-T, whole-body MRI unit (Magnetom Avanto, Siemens). An 18-G intravenous cannula was placed in the antecubital vein before prone positioning of patients on the MR table. Bilateral breast MRI was performed with a dedicated four-channel breast coil.

DCE-MRI data were obtained using multisection, T1-weighted, 3D vibe sequences with fat suppression before, during, and after IV bolus injection of gadoversetamide (Optimark); $0.1 \mathrm{mmol} / \mathrm{kg}$ of body weight of contrast was given at a rate of $1.5 \mathrm{ml} / \mathrm{s}$ followed by $10 \mathrm{ml}$ of saline flush at a rate of $1.5 \mathrm{ml} / \mathrm{s}$. A total of 30 measurements were taken, two before contrast, and the remaining ones during and after contrast injection.

Post-processing of dynamic contrast perfusion data was done by the vendor's Tissue $4 \mathrm{D}$ software.

Tumour volume was calculated by adding the volumes of individual slices, calculated as follows:

Volume $=$ area of lesion $\times($ thickness of slice + interstice gap $)$ 
After surgical resection, a pathological examination of the specimens was done and compared with post-treatment MRI parameters.

A cut-off value of $50 \%$ volume change was used as a criterion to classify treatment response. Responders showed at least a $50 \%$ decrease in volume, while nonresponders showed less than $50 \%$ decrease in tumour volume.

Pathological grading was done according to the criteria of Sataloff [5]. Grade A and B (more than 50\% therapeutic effect) were given to responders, while Grade $\mathrm{C}$ and $\mathrm{D}$ (less than $50 \%$ therapeutic effect) were given to non-responders.

We studied the perfusion parameters, ADC values, and tumour volume on MRI at baseline (first MRI), after two cycles (second MRI), and after completion of NACT (third MRI). After the last course of chemotherapy, all the selected patients underwent mastectomy or breast conserving therapy according to the standard protocols at our institute.

The pretreatment MRI parameters, i.e. $K^{\text {trans }}, K^{e p}, V_{e}$, IAUC, ADC value, and tumour volume, were then correlated with the post-chemotherapy values. A decrease in tumour diameter of the pathological specimen was compared with the initial baseline scan, and grading according to the Sataloff criteria was done.

Data were analysed using SPSS, version 17.0, for Windows. The means $( \pm$ SD) of all parameters at baseline and at completion of chemotherapy were calculated and compared by ANOVA. The changes in parameters were compared by paired $t$-tests for each category. Percentage changes in all MRI parameters were calculated and compared with treatment outcome/pathological specimen. $P<0.05$ was considered as statistically significant.

\section{Results}

Sixteen patients who met our inclusion criteria were enrolled. One patient died after the first cycle of chemotherapy (the cause was not reported), while another presented later with metastatic disease. Those two patients were excluded, and the final analysis was done in 14 patients.

Out of these 14 patients, nine received CAF-based NACT (cyclophosphamide, adriamycin, 5-fluorouracil), five received three cycles, four received four cycles, one received ECF-based NACT (cyclophosphamide, epirubicin, 5-fluorouracil - four cycles), and two patients received TAC-based CCT (docetaxel, adriamycin, 5-fluorouracil three cycles). One patient received one cycle of CAF followed by two cycles of TAC, and one patient received two cycles of docetaxel alone followed by two cycles of TAC followed by two cycles of gemcitabine and cisplatin-based NACT.

\section{Baseline characteristics}

The age range at presentation was 27 to 57 years. Fiftyseven per cent of patients had stage IIIB disease, $93 \%$
Table 1. Baseline characteristics

\begin{tabular}{|l|c|c|c|c|}
\hline No. & Age & $\begin{array}{c}\text { TNM } \\
\text { status }\end{array}$ & $\begin{array}{c}\text { Pre-treatment } \\
\text { stage }\end{array}$ & $\begin{array}{c}\text { Initial volume } \\
\text { of tumor in } \mathrm{cm}^{3}\end{array}$ \\
\hline 1 & 45 & T3N1 & IIB & 41 \\
\hline 2 & 27 & T2N2 & IIB & 47.7 \\
\hline 3 & 42 & T4BN2 & IIIB & 107 \\
\hline 4 & 57 & T3N2 & IIIA & 42 \\
\hline 5 & 52 & T4BN2M0 & IIIB & 167 \\
\hline 6 & 46 & T4BN2 & IIIB & 140 \\
\hline 7 & 31 & T3N1 & IA & 141 \\
\hline 8 & 38 & T4BN2M0 & IIIB & 116 \\
\hline 9 & 44 & T3N1M0 & IIIa & 15 \\
\hline 10 & 57 & T4BN1M0 & IIIB & 91 \\
\hline 11 & 38 & T4BN1M0 & IIB & 9.023 \\
\hline 12 & 45 & T4BN1M0 & IIIB & 397 \\
\hline 13 & 50 & T4BN1M0 & IIIB & 27 \\
\hline 14 & 56 & T3N2M0 & IIIA & 92 \\
\hline
\end{tabular}

Table 2. Baseline lesion volume and dynamic contrast-enhanced MRI (DCE-MRI) parameters and apparent diffusion coefficient (ADC) (all values expressed in mean \pm SD)

\begin{tabular}{|l|c|c|}
\hline Parameter & Mean & SD \\
\hline Lesion volume $\left(\mathrm{cm}^{3}\right)$ & 102.34 & 98.75 \\
\hline DCE-MRI parameters & & \\
\hline$K^{\text {rrans }}\left(\mathrm{min}^{-1}\right)$ & 0.25 & 0.15 \\
\hline$K^{\text {ep }}\left(\mathrm{min}^{-1}\right)$ & 1.19 & 0.83 \\
\hline$V_{e}$ & 0.33 & 0.15 \\
\hline IAUC & 10.12 & 5.94 \\
\hline ADC value $\left(\mathrm{mm}^{2} / \mathrm{s}\right)$ & 789.43 & 146.09 \\
\hline
\end{tabular}

(13 patients) had infiltrating duct cell carcinoma (NOS), and $7 \%$ (1 patient) had papillary cell carcinoma on histology (Table 1 ).

\section{Baseline parameters}

At baseline, MRI lesion volume varied from $9.023 \mathrm{~cm}^{3}$ to $397 \mathrm{~cm}^{3}$ with the mean of $102.34 \mathrm{~cm}^{3}$. $K^{\text {trans }}$ varied from 0.04 to 0.55 with the mean of $0.25( \pm 0.15), K^{e p}$ varied from 0.522 to 3.353 with the mean of 1.19 ( \pm 0.83 ), $V_{e}$ varied from 0.127 to 0.938 with the mean of 0.33 $( \pm 0.15)$, IAUC varied from 2.242 to 21.43 with the mean of 10.12 ( \pm 5.94$)$, and ADC varied from 534 to 1026 with the mean value of $789( \pm 146)$ (Table 2).

\section{Response to NACT}

Radiological and pathological complete response (CR) was seen in four patients (Table 3). 
Table 3. Response to neoadjuvant chemotherapy

\begin{tabular}{|l|c|c|}
$\begin{array}{l}\text { Type } \\
\text { of response }\end{array}$ & $\begin{array}{c}\text { Radiological response } \\
\text { (no. of patients) }\end{array}$ & $\begin{array}{c}\text { Pathological response } \\
\text { (no. of patients) }\end{array}$ \\
\hline CR & 4 & 4 \\
\hline NR & 9 & 10 \\
\hline Total & 13 & 14 \\
\hline
\end{tabular}

$\mathrm{CR}$ - complete response, $\mathrm{NR}$ - no response

\section{Correlation of MRI parameters with radiological response}

First MRI parameters, i.e. volume, $K^{\text {trans }}, K^{e p}, V_{e}$, IAUC, and $\mathrm{ADC}$, were not predictive of the final response to NACT (Table 4). At the second MRI, the IAUC value was significantly lower in responders than in non-responders $(p=0.023)$. None of the other parameters predicted response to NACT (Table 5). At the third MRI, comparison between responders and non-responders was not possible because all patients who underwent the third MRI had CR.
When changes in MRI parameters between the first and the last MRI in non-responders were compared, the $K^{e p}$ value changed significantly $(p=0.033)$. When changes in MRI parameters between the first and the last MRI in responders were compared, only the ADC value changed significantly $(p=0.012)$ (Tables 6 and 7).

On comparison of percentage changes, from the first to the second MRI, regarding volume, DCE parameters, and $\mathrm{ADC}$ in non-responders, the volume increased by $8.7 \%(p=0.86), V_{e}$ by $23 \%(p=0.29)$, and ADC by $26.6 \%$ $(p=0.06)$. In contrast, $K^{\text {trans }}$ decreased by $35 \%(p=0.19)$, $K^{e p}$ by $50.4 \%(p=0.03)$, and IAUC by $5.58 \%(p=0.83)$ (Table 6). When percentage changes from the first to the second MRI in volume, DCE parameters, and ADC were compared in responders, the volume decreased by $49.5 \%(p=0.204), K^{\text {trans }}$ by $17.6 \%(p=0.638), K^{\text {ep }}$ by $42 \%(p=0.04)$, IAUC by $70 \%(p=0.111)$, and $V_{e}$ by $28 \%$ $(p=0.422)$. In contrast, ADC increased by $34.5 \%$ $(p=0.084)$ (Table 7).

Table 4. Comparison of MRI parameters and volume among responders and non responders

\begin{tabular}{|c|c|c|c|c|}
\hline & \multicolumn{2}{|c|}{ Responders (CR) } & Non-responders (NR) & $p$ value \\
\hline \multicolumn{5}{|l|}{$1^{\text {st }} M R I$} \\
\hline Lesion volume & \multicolumn{2}{|c|}{$84.14 \pm 47.42$} & $120.11 \pm 116.31$ & 0.570 \\
\hline \multicolumn{5}{|l|}{ DCE parameters } \\
\hline$K^{\text {trans }}\left(\min ^{-1}\right)$ & \multicolumn{2}{|c|}{$0.17 \pm 0.05$} & $0.28 \pm 0.17$ & 0.261 \\
\hline$K^{e p}\left(\mathrm{~min}^{-1}\right)$ & \multicolumn{2}{|c|}{$1.40 \pm 1.31$} & $1.17 \pm 0.63$ & 0.660 \\
\hline$V_{e}$ & \multicolumn{2}{|c|}{$0.32 \pm 0.16$} & $0.30 \pm 0.13$ & 0.775 \\
\hline IAUC & \multicolumn{2}{|c|}{$10.28 \pm 7.47$} & $9.49 \pm 5.71$ & 0.837 \\
\hline $\mathrm{ADC}\left(\mathrm{mm}^{2} / \mathrm{s}\right)$ & \multicolumn{2}{|c|}{$845 \pm 128.60$} & $762.44 \pm 161.44$ & 0.389 \\
\hline \multicolumn{5}{|l|}{$2^{\text {nd }} M R I$} \\
\hline Lesion volume & $42.45 \pm 44.59$ & $130.58 \pm 125.17$ & 0.207 & \\
\hline \multicolumn{5}{|l|}{ DCE parameters } \\
\hline$K^{\text {trans }}\left(\mathrm{min}^{-1}\right)$ & $0.14 \pm 0.11$ & $0.18 \pm 0.13$ & 0.619 & \\
\hline$K^{e p}\left(\mathrm{~min}^{-1}\right)$ & $0.81 \pm 1.01$ & $0.58 \pm 0.41$ & 0.553 & \\
\hline$V_{e}$ & $0.23 \pm 0.14$ & $0.37 \pm 0.15$ & 0.129 & \\
\hline IAUC & $3.07 \pm 2.01$ & $8.96 \pm 4.17$ & \multicolumn{2}{|c|}{0.023} \\
\hline $\mathrm{ADC}\left(\mathrm{mm}^{2} / \mathrm{s}\right)$ & $1138 \pm 347.46$ & $965.67 \pm 250.60$ & \multicolumn{2}{|c|}{0.328} \\
\hline
\end{tabular}

Table 5. Percent change of MRI parameters among non-responder patients from baseline to first MRI

\begin{tabular}{|l|c|c|c|c|}
\hline & $1^{\text {st }}$ MRI (mean \pm SD) & $2^{\text {nd }}$ MRI (mean \pm SD $)$ & \% change & $p$ value \\
\hline Lesion volume & $120.11 \pm 116.31$ & $130.58 \pm 125.17$ & 8.70 & 0.86 \\
\hline DCE parameters & \multicolumn{3}{|c|}{} \\
\hline$K^{\text {Krans }}\left(\mathrm{min}^{-1}\right)$ & $0.28 \pm 0.17$ & $0.18 \pm 0.13$ & -35 & 0.19 \\
\hline$K^{\text {ep }}\left(\mathrm{min}^{-1}\right)$ & $1.17 \pm 0.63$ & $0.58 \pm 0.41$ & -50.4 & 0.03 \\
\hline$V_{e}$ & $0.30 \pm 0.13$ & $0.37 \pm 0.15$ & 23.3 & 0.29 \\
\hline IAUC & $9.49 \pm 5.71$ & $8.96 \pm 4.17$ & -5.58 & 0.83 \\
\hline $\operatorname{ADC}\left(\mathrm{mm}^{2} / \mathrm{s}\right)$ & $762.44 \pm 161.44$ & $965.67 \pm 250.60$ & 26.6 & 0.06 \\
\hline
\end{tabular}


Table 6. Percentage change of MRI parameters from $1^{1 \text { st }}$ to $2^{\text {nd }}$ MRI among responders

\begin{tabular}{|c|c|c|c|c|}
\hline & $1^{\text {st }} \mathrm{MRI}($ mean \pm SD) & $2^{\text {nd }}$ MRI (mean \pm SD) & $\%$ change & $p$ value \\
\hline Lesion volume & $84.14 \pm 47.42$ & $42.45 \pm 44.59$ & -49.5 & 0.204 \\
\hline \multicolumn{5}{|c|}{ DCE parameters } \\
\hline$K^{\text {trans }}\left(\min ^{-1}\right)$ & $0.17 \pm 0.05$ & $0.14 \pm 0.11$ & -17.6 & 0.638 \\
\hline$K^{e p}\left(\min ^{-1}\right)$ & $1.40 \pm 1.31$ & $0.81 \pm 1.01$ & -42.1 & 0.04 \\
\hline$V_{e}$ & $0.32 \pm 0.16$ & $0.23 \pm 0.14$ & -28.12 & 0.422 \\
\hline IAUC & $10.28 \pm 7.47$ & $3.07 \pm 2.01$ & -70.1 & 0.111 \\
\hline $\mathrm{ADC}\left(\mathrm{mm}^{2} / \mathrm{s}\right)$ & $845 \pm 128.60$ & $1138 \pm 347.46$ & 34.7 & 0.084 \\
\hline
\end{tabular}

When percentage changes, from first to third MRI, regarding volume, DCE parameters, and ADC were compared in responders, volume decreased by $86 \%(p=0.10)$, $K^{\text {trans }}$ by $0.86 \%(p=0.93), K^{e p}$ by $88 \%(p=0.34)$, IAUC by $64 \%(p=0.15)$, and $V_{e}$ increased by $19 \%(p=0.28)$, and $\operatorname{ADC}$ by $86 \%(p=0.01)$.

\section{Prediction of pathological response}

Based on Sataloff criteria for pathological responses, four patients out of 14 were responders, and 10 were non-responders. None of the measured parameters predicted pathological response.

\section{Discussion}

\section{Volume}

Partridge et al. assessed the value of MRI measurements of breast tumour size for predicting recurrence-free survival (RFS) in 62 patients receiving neoadjuvant chemotherapy. The longest tumour diameter and volume were measured on MRI before and after one and four cycles of treatment. Final change in the MRI volume $(p=0.015)$ was more predictive than change in the diameter on MRI ( $p=0.077)$ or clinical examination $(p=0.27)$ [6]. In our study, we used a $50 \%$ reduction in volume as a criterion for response assessment; however, it could not predict the final pathological or radiological response.

In a study by Cho et al. (2013) 48 women with breast cancer were enrolled and treated with an anthracycline taxane regimen. Tumour size and volume, PRM characteristics, and pharmacokinetic parameters ( $K^{\text {trans }}, K^{e p}$, and $V_{e}$ ) on MR images were assessed and compared according to the pathologic responses. Six of 48 (12\%) patients showed complete response on pathology (pCR) and 42 (88\%) showed non-pathologic CR (npCR). Thirty-eight (79\%) patients showed good radiological response, and $10(21 \%)$ showed a minor response. No difference in tumour size, tumour volume, or pharmacokinetic parameters was found between the groups. In our study, volume reduction from baseline to the first MRI was $49 \%$ ( $p=0.204)$, and $86 \%(p=0.10)$ from baseline to the third
Table 7. Percentage change in volume, DCE parameters and apparent diffusion coefficient $(A D C)$ in responder patients from 1 st to $3^{\text {rd }} M R I(N=3)$

\begin{tabular}{|c|c|c|c|c|}
\hline & $\begin{array}{c}1^{\text {st }} \text { MRI } \\
(\text { mean } \pm \text { SD) }\end{array}$ & $\begin{array}{c}3^{\text {rd }} \text { MRI } \\
(\text { mean } \pm \text { SD) }\end{array}$ & $\begin{array}{c}\% \\
\text { Change }\end{array}$ & $p$ value \\
\hline Volume $\left(\mathrm{cm}^{3}\right)$ & $65.57 \pm 20.78$ & $8.80 \pm 3.37$ & -86.57 & 0.10 \\
\hline$K^{\text {trans }}\left(\min ^{-1}\right)$ & $0.15 \pm 0.03$ & $0.15 \pm 0.09$ & -0.86 & 0.93 \\
\hline$K^{e p}\left(\min ^{-1}\right)$ & $1.59 \pm 0.89$ & $0.19 \pm 0.03$ & -88 & 0.34 \\
\hline$V_{e}$ & $0.34 \pm 0.11$ & $0.41 \pm 0.06$ & 19.34 & 0.28 \\
\hline IAUC & $11.10 \pm 5.15$ & $3.95 \pm 1.69$ & -64.38 & 0.15 \\
\hline $\mathrm{ADC}\left(\mathrm{mm}^{2} / \mathrm{s}\right)$ & $893 \pm 60.51$ & $1666 \pm 163.81$ & 86.56 & 0.01 \\
\hline
\end{tabular}

MRI in responders, and it increased by $8 \%(p=0.86)$ in non-responders from baseline to the first MRI; however, the changes were not statistically significant and therefore that study is concordant with our study [7].

Pickles et al. (2005) assed response to NACT with DCE-MRI in 68 patients with histologically proven breast cancer. MRI was performed at the initiation of NACT, early during treatment and after the final cycle of NACT. Following treatment, 48 patients were classified as responders and 20 as non-responders based on total tumour volume reduction. In their study, pre-treatment volume was higher in responders, with a mean of $34.09 \mathrm{~cm}^{3}$, as compare to non-responders, who had a volume of $29.22 \mathrm{~cm}^{3}$. Tumour volume changes between pre-treatment and early treatment time points demonstrated differences between responders and non-responders, with the percentage change being most significant $(0.813 \%, p<0.001)$. In our study, pre-treatment tumour volume was higher in non-responders. It was $84.14 \pm 42.42 \mathrm{~cm}^{3}$ at baseline and decreased to $42.42 \pm 44.49 \mathrm{~cm}^{3}$ after two cycles ( $49 \%$, $p=0.204)$ in responders, and in non-responders baseline tumour volume was $120.11 \pm 116.31 \mathrm{~cm}^{3}$ and increased to $130.58 \pm 125.17 \mathrm{~cm}^{3}$ after two cycles of NACT $(8 \%$, $p=0.86$ ). Therefore, our study found similar changes in responders and non-responders, but the change was not statistically significant [8].

In 2013, Nadrljansky et al. studied 66 patients before the first treatment cycle, after the second cycle, and upon completion of neoadjuvant chemotherapy, with regard to the largest tumour diameter, tumour volume, post-con- 
trast enhancement, and tumour regression pattern. The average target lesion volume was initially $32.2 \mathrm{~cm}^{3} \mathrm{com}$ pared to $17.1 \mathrm{~cm}^{3}$ after the two cycles $(p<0.01)$ and $4.9 \mathrm{~cm}^{3}$ after completion of NACT $(p<0.001)$. The average target lesion volume decreased by $46.9 \%$ after two cycles of NACT, which was below the threshold limit of $65 \%$ volume decrease for response, and it decreased by $84.8 \%$ upon completion of NACT. In responders $(n=27)$ the lesion size upon completion of neoadjuvant chemotherapy was significantly smaller than in non-responders (1.5 \pm 0.6 vs. $3.2 \pm 0.9 \mathrm{~cm} ; p<0.001$ ), as was the volume (1.2 vs. $11.0 \mathrm{~cm}^{3} ; p<0.001$ ) [9]. Similarly, in our study the initial lesion size in responders was $65 \mathrm{~cm}^{3}$ as compared to 8.8 $\mathrm{cm}^{3}$ at completion of NACT. The average target volume decreased by $49 \%(p=0.21)$ after two cycles of NACT, which was near to the threshold limit of 50\% for response, and it decreased by $86 \%(p=0.857)$ after completion of NACT. In non-responders, the volume increased by $8 \%(p=0.86 \%)$, although this change was not statistically significant.

Padhani et al. performed a prospective study to document changes in contrast agent kinetics in patients with primary breast cancer treated with NACT. Twenty-five patients underwent DCE-MRI examination before initiating treatment, after the first cycle and then after the second cycle of NACT. After the second cycle of NACT, in five of six non-responders the tumour size increased, compared with a decrease in eight out of nine responders. Reductions in transfer constant range were also observed in responders with regard to both clinical and pathological assessments. They concluded that reductions in the volume of the primary tumour best predict clinical and pathologic response of breast cancer after one cycle of neoadjuvant chemotherapy [3]. Our study showed similar trends with reduction in tumour volume in responders $(49 \%, p=0.204)$ and an increase in non-responders (8\%, $p=0.86 \%)$ after two cycles of NACT, although this difference was not significant statistically.

\section{Tissue parametric imaging}

In 2002, 21 patients with locally advanced breast cancer on NACT were studied by Wasser et al. [10], who evaluated changes in both size and contrast enhancement of breast tumours during neoadjuvant chemotherapy using dynamic MRI. A reduction in tumour size after chemotherapy of more than $25 \%$ was associated with a decrease in both parameters ( $K^{e p}, p<0.002$, amplitude, $\left.p<0.006\right)$. $K^{e p}$ began to drop after the first cycle of chemotherapy. A clear reduction in tumour size was only noted after the third cycle. In our study, in responders there was a statistically significant reduction in $K^{e p}$ with reduction in tumour size. A significant reduction in $K^{\text {ep }}$ values was also seen in non-responders, although the tumour volume increased. This finding is in disagreement with the study of Wasser et al., in which changes were significant only in responders.
In 2007, Yu et al. [4] investigated 29 patients with invasive breast cancer. Tumour size, $K^{\text {trans }}$, and $K^{\text {ep }}$ were used to predict final clinical response to NACT. Doxorubicin and cyclophosphamide (AC) chemotherapy was given every two weeks. Significant correlation was seen between early changes in tumour size and both $K^{\text {trans }}$ and $K^{e p}$. Early tumour size change on MRI after one cycle was found to be a better response predictor than either $K^{\text {trans }}$ or $K^{e p}$. In contrast to this study, our study showed significant correlation between early changes in $K^{e p}$, but the change was significant among non-responders as well. In contrast to that study, no significant correlation was seen between early change in volume and $K^{\text {trans }}(p=0.21$ and $p=0.638$, respectively).

Pickles et al. noted that both $K^{\text {trans }}$ and $K^{e p}$ showed a significant reduction in responders between the pre-treatment and early treatment time points. In contrast, a significant increase in $V_{e}$ was seen in non-responders. Contrary to our study, no significant reduction in $K^{\text {trans }}(p=0.638)$ was seen in pre-treatment and early treatment endpoints in responders. $K^{\text {ep }}$ decreased significantly in both responders and non-responders. A 23\% increase in $V_{e}$ was seen among non-responders; however, it was not statistically significant $(p=0.291)$ [11].

In 2007, Thukral et al. [12] retrospectively compared three dynamic contrast-enhanced MRI examinations to determine the parameters or a combination of parameters that were most strongly associated with changes in tumour microvasculature during treatment with bevacizumab alone and bevacizumab plus chemotherapy in patients with inflammatory or locally advanced breast cancer. Twenty-one women were enrolled. In 19 patients, using a whole-tumour region of interest, the authors observed a significant decrease in the median values of the three following parameters measured from baseline to the first cycle: forward transfer rate constant $\left(K^{\text {trans }}\right)(-34 \%$ relative change, $p=0.003)$, backflow compartmental rate constant extravascular and extracellular to constant $\left(K^{e p}\right)$ $(-15 \%$ relative change, $p<0.001)$, and integrated area under the gadolinium concentration curve (IAUGC) at 180 seconds $(-23 \%$ relative change, $p=0.009)$. In our study, no significant decrease in $K^{\text {trans }}(-17.6 \%$ relative change, $p=0.638), V_{e}(-28.1 \%$ relative change, $p=0.422)$, IAUC $(-70.1 \%$ relative change, $p=0.111)$ was seen after the third MRI. Only the decrease in $K^{e p}$ was significant $(-42.1 \%$ relative change, $p=0.04)$.

De Bazelaire et al. [13] performed a retrospective study in 24 women with locally advanced breast cancer. MRI was performed before NACT and after eight cycles of treatment. This was followed by surgery less than four weeks after the last course of chemotherapy. Changes in kinetic parameters after treatment were compared with final pathologic response graded as non-responding ( $<50 \%$ therapeutic effect), partially responding $(>50 \%$ therapeutic effect), and completely responding. Changes in $V_{e}$ and $K^{\text {trans }}$ were significantly different between non 
$(n=11)$, partial $(n=7)$, and complete $(n=6)$ responders $(p=0.0092$ and $p=0.0398$, respectively). A decrease in $V$ of more than $-72 \%$ and more than $-84 \%$ for $K^{\text {trans }}$ resulted in $73 \%$ sensitivity for identifying non-responders (specificity $92 \%$ and $77 \%$, respectively). A decrease in $V$ of more than $-87 \%$ helped to identify complete responders. In contrast, our study found no significant difference in the parameters when baseline scan was compared with the final pathological response between responders and non-responders ( $K^{\text {trans }} 0.15 \pm 0.05$ vs. $0.27 \pm 0.16, K^{\text {ep }} 1.59$ \pm 1.53 vs. $1.08 \pm 0.61, V_{e} 0.34 \pm 0.19$ vs. $0.32 \pm 0.15$, IAUC $11.10 \pm 8.93$ vs. $9.86 \pm 5.44$ in responders and non-responders, respectively; with $p=0.242,0.365,0.844$, and 0.763 for $K^{\text {trans }}, K^{e p}, V$, and IAUC, respectively). In addition, $V_{e}$ increased by $19 \%$ between the first and the final MRI among responders $(p=0.28)$. On the second MRI, $V_{e}$ decreased by $28 \%(p=0.422)$ among responders and increased by $23 \%(p=0.291)$ in non-responders; the changes were statistically significant.

Johansen et al. [14] evaluated DCE-MRI in patients scheduled for NACT $(n=24)$ before and after the first treatment cycle. Clinical response was evaluated after completed NACT. Relative signal intensity (RSI) and AUC were calculated from DCE-curves and compared with clinical treatment response. They found that RSI and AUC were reduced after only one cycle of NAC in patients with clinical treatment response $(p=0.02$ and $p=0.08)$.

Our results are consistent with that study, but after two cycles of NACT, IAUC was a significant predictor of response to chemotherapy. The mean IAUC value in responders was $3.07 \pm 2.01$ and $8.96 \pm 4.17$ in non-responders $(p=0.023)$. Although our study group was small, IAUC showed an early change in responding patients. It was a good predictor of response; however, comparison at the third MRI was not possible because none of the non-responders underwent the third MRI.

\section{Diffusion-weighted imaging}

Park et al. [15], in a retrospective study (2010), evaluated 53 consecutive women with invasive breast cancer, who underwent NACT. Both DWI MR imaging and DCEMRI were performed at $1.5 \mathrm{~T}$ before and after NACT prior to surgery. Pre-treatment ADCs and percentage increases in ADC after chemotherapy in responders and non-responders were compared. Patients with reduced tumour diameter of at least $30 \%$ after chemotherapy at DCE-MRI were defined as responders. After chemotherapy, 36 patients were classified as responders, and 17 were classified as non-responders. The pre-treatment mean ADC of responders was significantly lower than that of non-responders. Furthermore, the mean percentage ADC increase of responders (47.9\%) was higher than that of non-responders (18.1\%). The best pre-treatment ADC cutoff for differentiation between responders and non-responders was $1.17 \times 103 \mathrm{~mm}^{2} / \mathrm{s}$.
In our study, the ADC values at baseline were higher $(845 \pm 128)$ in than in non-responders $(762.44 \pm 161.44$, $p=0.389$ ). This finding is in disagreement with the study by Park et al. The ADC value in responding patients increased from $845 \pm 128$ to $1138 \pm 347$ at the second MRI $(34 \%, p=0.084)$ and then to $1666 \pm 284$ at the third MRI $(86.6 \%)$. At the third MRI, this change was significant $(p=0.01)$. In contrast, the change in ADC values in non-responders was not significant $(762.44 \pm 161.44$ at the first MRI to $965.67 \pm 250.60$ at the second MRI). This aspect of our study is in line with the study by Park et al.

Kawamura et al. [16] evaluated 11 breast cancer patients (12 lesions) scheduled for NACT. The patients were examined with MRI prior to and after the first and fourth course of anthracycline-containing chemotherapy and after subsequent taxane-containing chemotherapy. Lesions were divided into two types - the mass type and non-mass type, based on contrast MRI performed prior to chemotherapy. Among eight patients with mass type lesions, six were responders and two were non-responders. Responders showed either an increased apparent diffusion coefficient (ADC) or volume reduction after the first course of NACT, whereas non-responders showed neither $(p<0.005)$. Of the four patients with non-mass type lesions, two were responders and two were non-responders. Changes in ADC or volume after the first course of NACT may indicate chemosensitivity of mass type breast cancer lesions.

In our study, responders also showed increased ADC $(34 \%, p=0.084)$ and volume reduction $(49 \%, p=0.204)$, but it was not statistically significant after two cycles of NACT, whereas non-responders showed a $26 \%$ decrease in $\operatorname{ADC}(p=0.06)$ with an $8.7 \%$ increase in volume $(p=0.857)$.

Jensen et al. [17] evaluated DWI and DCE-MRI in 15 breast cancer patients, performed before and after one cycle of NACT. MRI tumour diameter and volume, apparent diffusion coefficient (ADC), and kinetic parameters $\left(K^{\text {trans }}\right.$ and $\left.V_{e}\right)$ were evaluated. The reliability of ADC before NACT was assessed. The reliability of ADC values was high, with an intraclass correlation coefficient of 0.84 $(p=0.001)$. After one cycle of NACT, MRI tumour diameter $(8 \%, p=0.005)$ and tumour volume $(30 \%, p=0.008)$ were reduced in all patients, while the mean $\mathrm{ADC}$ values increased $\left(0.12 \mathrm{~mm}^{2} / \mathrm{s}, p=0.008\right)$. Similarly, in our study, after two cycles of NACT, in responders, tumour volume decreased from $84.14 \pm 47.22$ to $42.45 \pm 44.59$ (49\%, $p=0.204)$, while the mean ADC value increased from 845 \pm 128 to $1138 \pm 347$ ( $34 \%, p=0.084)$, but these changes were not statistically significant.

\section{Pathological response}

In the study by Loo et al. [18], dynamic contrast-enhanced MRI was performed before chemotherapy and after two chemotherapy cycles in 54 breast cancer patients. Imaging was correlated with final pathology. The change in the 
largest diameter of late enhancement during chemotherapy was the single most predictive MRI characteristic for tumour response in a multivariate analysis. A decrease in the largest diameter of less than 25\% during chemotherapy was most indicative of residual tumour at final pathology. Using this criterion, the fraction of unfavourable responders indicated by MRI was $41 \%(22 / 54)$. Approximately half $(44 \%, 14 / 32)$ of the patients who showed favourable response at MRI achieved complete remission at pathology. Conversely, 95\% (21/22) of patients who showed unfavourable response at MRI had residual tumour at pathology.

Likewise, in our study the concordance between the pathological and radiological response rate was good, with four out of the four radiological responders showing pathological CR. Thus, the coherence between the two modalities was found to be good (100\%). However, none of the parameters predicted pathological response when baseline MRI parameters including volume, $K^{\text {trans }}(p=0.489)$, $K^{e p}(p=0.242), V_{e}(p=0.365)$, IAUC $(p=0.844)$, and ADC $(p=0.175)$ were compared with the final pathological response among responders and non-responders.

In 2008, Ah-See et al. [19] examined 37 patients with breast cancer, who were to receive six cycles of ECF-based chemotherapy (epirubicin, cisplatin, and 5-fluorouracil). Their patients underwent DCE-MRI studies before the initiation of chemotherapy and after two cycles of therapy. Twenty-eight patients were evaluated with regard to response (19 clinical responders and nine non-responders). Changes in the DCE-MRI kinetic parameters $K^{\text {trans }}$ and $K^{e p}$ were significantly correlated with both final clinical and pathologic response. The change in $K^{\text {trans }}$ was the best predictor of pathologic response, correctly identifying $94 \%$ of non-responders and $73 \%$ of responders. Interestingly, the change in MRI-derived tumour size did not predict pathologic response. In our study, $K^{\text {trans }}, K^{e p}, V_{e}$, and IAUC were not predictive of the final pathologic response $(p=0.242, p=0.265, p=0.844, p=0.763$, respectively). However, in line with that study, the change in MRIderived tumour volume did not predict pathologic response $(p=0.489)$.

In a recent review by Galbán et al., of three different prospective clinical trials [20], in which a total of 39 patients with LABC underwent DW-MRI prior to and at 3-7 days, 8-11 days, and 35 days post-treatment initiation, the mean tumour ADC values generated from patient test-retest examinations were found to be very reproducible. A parametric response map was calculated and was found to be predictive of the outcome at day 35 (AUC = $0.770, p=0.05)$.

In another study by Huang et al. [21], DCE-MRI data acquired in one centre from 10 patients with breast cancer before and after the first cycle of neoadjuvant chemotherapy were reviewed with 12 software tools. Nearly all tools provided early prediction of therapy response using the mean tumour $K^{\text {trans }}$ and $K^{e p}\left(=K^{\text {trans }} / V_{e}\right.$, intravasation rate constant) after the first therapy cycle. The authors concluded that the algorithm parameter variation did not significantly affect the utility of DCE-MRI for assessing therapy response.

The small study group consisting of 14 patients was a limiting factor of our study and thus the results need to be interpreted in a larger study for validation. Moreover, the chemotherapy regimens used in our study were varied, and the number of chemotherapy cycles was not uniform.

Long acquisition times and uncomfortable positioning of patients was also a significant issue, with eight of the 14 patients complaining of the long and uncomfortable positioning, although none refused to participate.

\section{Conclusions}

DCE-MRI was performed in 14 patients to search for predictors of response to NACT in locally advanced breast cancer. MRI was done at baseline, after two cycles, and finally after completion of chemotherapy. At the second MRI examination, IAUC was found to be significantly lower in responders than in non-responders. The decrease in $K^{e p}$ values was statistically significant in both responders and non-responders. An increase in ADC values was statistically significant from the first to the third MRI in responders, and it also increased in non-responders, albeit not significantly. In our study, IAUC and ADC were the only parameters that could reliably differentiate responders from non-responders after two and three cycles of chemotherapy.

\section{Conflict of interest}

The authors report no conflict of interest.

\section{References}

1. O’Flynn EA, DeSouza NM. Functional magnetic resonance: biomarkers of response in breast cancer. Breast Cancer Res 2011; 13: 204.

2. O'Connor JP, Jackson A, Parker GJ, et al. DCE-MRI biomarkers in the clinical evaluation of antiangiogenic and vascular disrupting agents. Br J Cancer 2007; 96: 189-195.
3. Padhani AR, Hayes C, Assersohn L, et al. Prediction of clinicopathologic response of breast cancer to primary chemotherapy at contrast-enhanced MR imaging: initial clinical results. Radiology 2006; 239: 361-374.

4. Yu HJ, Chen JH, Mehta RS, et al. MRI measurements of tumor size and pharmacokinetic parameters as early predictors of response 
in breast cancer patients undergoing neoadjuvant anthracycline chemotherapy. J Magn Reson Imaging 2007; 26: 615-623.

5. Sataloff DM, Mason BA, Prestipino AJ, et al. Pathologic response to induction chemotherapy in locally advanced carcinoma of the breast: a determinant of outcome. J Am Coll Surg 1995; 180: 297-306.

6. Partridge SC, Gibbs JE, Lu Y, et al. MRI measurements of breast tumor volume predict response to neoadjuvant chemotherapy and recurrence-free survival. AJR Am J Roentgenol 2005; 184: 1774-1781.

7. Cho N, Im SA, Park IA, et al. Breast cancer: early prediction of response to neoadjuvant chemotherapy using parametric response maps for MR imaging. Radiology 2014; 272: 385-396.

8. Pickles MD, Lowry M, Manton DJ, et al. Role of dynamic contrast enhanced MRI in monitoring early response of locally advanced breast cancer to neoadjuvant chemotherapy. Breast Cancer Res Treat 2005; 91: 1-10.

9. Nadrljanski MM, Miloševic ZC, Plešinac-Karapandžic V, et al. MRI in the evaluation of breast cancer patient response to neoadjuvant chemotherapy: predictive factors for breast conservative surgery. Diagn Interv Radiol 2013; 19: 463-470.

10. Wasser K, Klein SK, Fink C, et al. Evaluation of neoadjuvant chemotherapeutic response of breast cancer using dynamic MRI with high temporal resolution. Eur Radiol 2003; 13: 80-87.

11. Pickles MD, Lowry M, Manton DJ, et al. Role of dynamic contrast enhanced MRI in monitoring early response of locally advanced breast cancer to neoadjuvant chemotherapy. Breast Cancer Res Treat 2005; 91: 1-10.

12. Thukral A, Thomasson DM, Chow CK, et al. Inflammatory breast cancer: dynamic contrast-enhanced MR in patients receiving bevacizumab - initial experience. Radiology 2007; 244: 727-735.

13. De Bazelaire C, Calmon R, Thomassin I, et al. Accuracy of perfusion MRI with high spatial but low temporal resolution to assess invasive breast cancer response to neoadjuvant chemotherapy: a retrospective study. BMC Cancer 2011; 11: 361.
14. Johansen R, Jensen LR, Rydland J, et al. Predicting survival and early clinical response to primary chemotherapy for patients with locally advanced breast cancer using DCE-MRI. J Magn Reson Imaging 2009; 29: 1300-1307.

15. Park SH, Moon WK, Cho N, et al. Comparison of diffusion-weighted MR imaging and FDG PET/CT to predict pathological complete response to neoadjuvant chemotherapy in patients with breast cancer. Eur Radiol 2012; 22: 18-25.

16. Kawamura M, Satake H, Ishigaki S, et al. Early prediction of response to neoadjuvant chemotherapy for locally advanced breast cancer using MRI. Nagoya J Med Sci 2011; 73: 147-156.

17. Jensen LR, Garzon B, Heldahl MG, et al. Diffusion-weighted and dynamic contrast-enhanced MRI in evaluation of early treatment effects during neoadjuvant chemotherapy in breast cancer patients. J Magn Reson Imaging 2011; 34: 1099-1109.

18. Loo CE, Teertstra HJ, Rodenhuis S, et al. Dynamic contrast-enhanced MRI for prediction of breast cancer response to neoadjuvant chemotherapy: initial results. AJR Am J Roentgenol 2008; 191: 1331-1338.

19. Ah-See ML, Makris A, Taylor NJ, et al. Early changes in functional dynamic magnetic resonance imaging predict for pathologic response to neoadjuvant chemotherapy in primary breast cancer. Clin Cancer Res 2008; 14: 6580-6589.

20. Galbán CJ, Ma B, Malyarenko D, et al. Multi-site clinical evaluation of DW-MRI as a treatment response metric for breast cancer patients undergoing neoadjuvant chemotherapy. PLoS One 2015; 10: e0122151.

21. Huang W, Li X, Chen Y, et al. Variations of dynamic contrast-enhanced magnetic resonance imaging in evaluation of breast cancer therapy response: a multicenter data analysis challenge. Transl Oncol 2014; 7 : 153-166. 